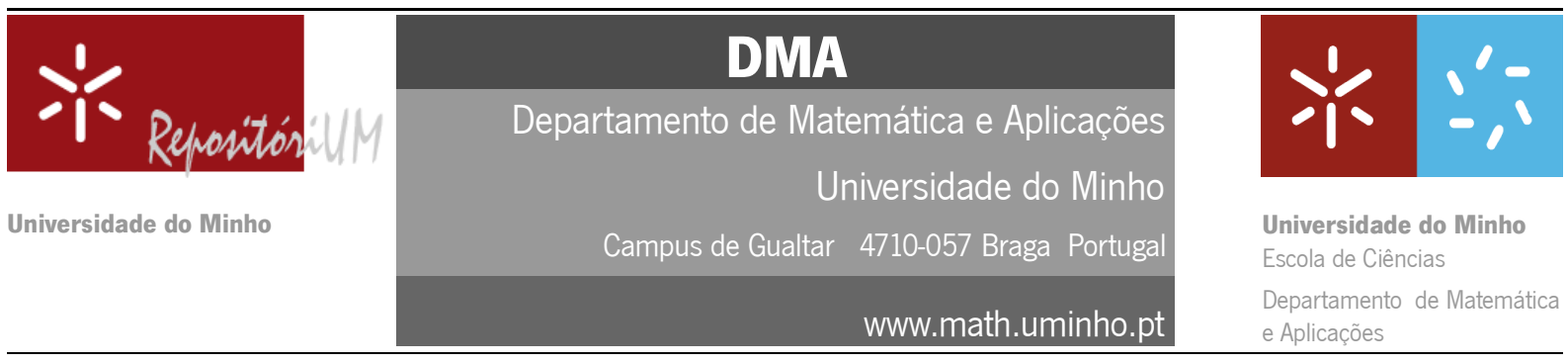

\title{
Clifford Analysis between continuous and discrete
}

\author{
H.R. Malonek ${ }^{a}$ \\ M.I. Falcão ${ }^{b}$ \\ ${ }^{a}$ Departamento de Matemática, Universidade de Aveiro, Portugal \\ ${ }^{b}$ Departamento de Matemática e Aplicações, Universidade do Minho, Portugal
}

\section{Information}

Keywords:

Homogeneous monogenic polynomials, quaternions, Appell sets.
Original publication:

AIP Conference Proceedings, 2008, 1048, 682-685

DOI: $10.1063 / 1.2991019$

link.aip.org/link/?APCPCS/1048/682/1

\begin{abstract}
Some decades ago D. Knuth et al. have coined concrete mathematics as the blending of CONtinuous and disCRETE math, taking into account that problems of standard discrete mathematics can often be solved by methods based on continuous mathematics together with a controlled manipulation of mathematical formulas. Of course, it was not a new idea, but due to the ongoing emergence of computer aided algebraic manipulation tools of that time it emphasized their use for elegant solutions of old problems or even the detection of new important relationships. Our aim is to show that the same philosophy can be successfully applied to Clifford Analysis by taking advantages of its inherent non-commutative algebra to obtain results or develop methods that are different from other ones. In particular, we determine new binomial sums by using a hypercomplex generating function for a special type of monogenic polynomials and develop an algorithm for the determination of their scalar and vector part which illustrates well the differences to the corresponding complex case.
\end{abstract}

\section{Introduction}

The book [1] with the extremely short title $\mathbf{A}=\mathbf{B}$ opens with the citation of an exercise from [2]:

"Develop computer programs for simplifying sums that involve binomial coefficients."

Another book [3] with the curious title generatingfunctionology is not a compendium for scientologist, nevertheless it is an exciting excursion into the theory of generating functions and holds many dangers for becoming a follower of a really scientific sect. In an expert review of this book one can read: 


\begin{abstract}
"Generating functions are a bridge between discrete mathematics, on the one hand, and continuous analysis (particularly complex variable theory) on the other. It is possible to study them solely as tools for solving discrete problems. As such there is much that is powerful and magical in the way generating functions give unified methods for handling such problems."
\end{abstract}

We mention these facts because both books are dealing with subjects in the core of concrete mathematics (in the sense of $\mathrm{D}$. Knuth $\mathrm{t}$ al. [4]): binomial sums and generating functions.

The importance of complex variable theory as quoted in the review leads naturally to the question about contributions of Clifford Analysis to this area. Indeed, the fact that Clifford Analysis relies essentially on methods from complex function theory, in particular on power series and related methods, suggests an affirmative answer to this question. By taking advantages of its inherent non-commutative algebra it is possible to obtain results or develop methods that are different from those in a commutative setting. As an example we will show how a special hypercomplex generating functions can be used for determining new binomial sums.

\title{
2 Basic Notations
}

Let $\left\{e_{1}, e_{2}, \cdots, e_{n}\right\}$ be an orthonormal base of the Euclidean vector space $\mathbb{R}^{n}$ with a product according to the multiplication rules $e_{k} e_{l}+e_{l} e_{k}=-2 \delta_{k l}, k, l=1, \cdots, n$, where $\delta_{k l}$ is the Kronecker symbol. This non-commutative product generates the $2^{n}$-dimensional Clifford algebra $C l_{0, n}$ over $\mathbb{R}$ and the set $\left\{e_{A}: A \subseteq\right.$ $\{1, \cdots, n\}\}$ with $e_{A}=e_{h_{1}} e_{h_{2}} \cdots e_{h_{r}}, 1 \leq h_{1} \leq \cdots \leq h_{r} \leq n, e_{\emptyset}=e_{0}=1$, forms a basis of $C l_{0, n}$. The real vector space $\mathbb{R}^{n+1}$ will be embedded in $C l_{0, n}$ by identifying the element $\left(x_{0}, x_{1}, \cdots, x_{n}\right) \in \mathbb{R}^{n+1}$ with the element $x=x_{0}+\underline{x}$ of the algebra, where $\underline{x}=e_{1} x_{1}+\cdots+e_{n} x_{n}$. The conjugate of $x$ is $\bar{x}=x_{0}-\underline{x}$ and the norm $|x|$ of $x$ is defined by $|x|^{2}=x \bar{x}=\bar{x} x=x_{0}^{2}+x_{1}^{2}+\cdots+x_{n}^{2}$. Denote by $\omega(x)=\frac{\underline{x}}{|\underline{x}|} \in S^{n}$, where $S^{n}$ is the unit sphere in $\mathbb{R}^{n}$.

In what follows we consider $C l_{0, n}$-valued functions defined in some open subset $\Omega \subset \mathbb{R}^{n+1}$, i.e., functions of the form $f(z)=\sum_{A} f_{A}(z) e_{A}$, where $f_{A}(z)$ are real valued. We suppose that $f$ is hypercomplex differentiable in $\Omega$ in the sense of [5], [6], i.e. has a uniquely defined areolar derivative $f^{\prime}$ in each point of $\Omega$. Then $f$ is real differentiable (even real analytic) and $f^{\prime}$ can be expressed in terms of the partial derivatives with respect to $x_{k}$ as $f^{\prime}=1 / 2\left(\partial_{0}-\partial_{\underline{x}}\right) f$, where $\partial_{0}:=\frac{\partial}{\partial x_{0}}, \quad \partial_{\underline{x}}:=e_{1} \frac{\partial}{\partial x_{1}}+\cdots+e_{n} \frac{\partial}{\partial x_{n}}$. If now $D:=\partial_{0}+\partial_{\underline{x}}$ is the usual generalized Cauchy-Riemann differential operator, then, obviously $f^{\prime}=1 / 2 \bar{D} f$. Since in [5] it has been shown that a hypercomplex differentiable function belongs to the kernel of $D$, i.e. satisfies the property $D f=0$ ( $f$ is a monogenic function in the sense of Clifford Analysis), then it follows that in fact $f^{\prime}=\partial_{0} f$ like in the complex case. For our purpose we use monogenic polynomials in terms of the hypercomplex monogenic variables $z_{k}=x_{k}-x_{0} e_{k}=-\frac{x e_{k}+e_{k} x}{2}, k=1,2, \cdots, n$, which leads to generalized powers of degree $n$ that are by convention symbolically written as $z_{1}^{\nu_{1}} \times \cdots z_{n}^{\nu_{n}}$ and defined as an $n$-nary symmetric product by $z_{1}^{\nu_{1}} \times \cdots z_{n}^{\nu_{n}}=\frac{1}{n !} \sum_{\pi\left(i_{1}, \ldots, i_{n}\right)} z_{i_{1}} \cdots z_{i_{n}}$, where the sum is taken over all permutations of $\left(i_{1}, \ldots, i_{n}\right)$, (see [6]).

\section{Binomial sums and an Appell set of Polynomials in $\mathbb{R}^{n+1}$}

An easy example for the relationship between binomial sums and complex variables is the following. Consider positive integer powers $z^{k}=(x+i y)^{k}$, develop them as binomial and then specify the values of $x$ and $y$ as desired. At the end compare on both sides the real and the imaginary part. For $k \equiv 0,1,2,3(\bmod 4)$, and $x=y=1,(1+i)^{4 l}=(2 i)^{2 l}=(-4)^{l},(1+i)^{4 l+1}=(-4)^{l}(1+i)$ etc. it results, for example, in

$$
\begin{aligned}
& \left(\begin{array}{c}
4 l+1 \\
0
\end{array}\right)-\left(\begin{array}{c}
4 l+1 \\
2
\end{array}\right)+\left(\begin{array}{c}
4 l+1 \\
4
\end{array}\right)-\cdots+\left(\begin{array}{c}
4 l+1 \\
4 l
\end{array}\right)=(-4)^{l} \\
& \left(\begin{array}{c}
4 l+3 \\
0
\end{array}\right)-\left(\begin{array}{c}
4 l+3 \\
2
\end{array}\right)+\left(\begin{array}{c}
4 l+3 \\
4
\end{array}\right)-\cdots-\left(\begin{array}{c}
4 l+3 \\
4 l+1
\end{array}\right)=(-1)^{l+1} 2^{2 l+1} \quad \text { etc. }
\end{aligned}
$$

The remarks in the previous section on the non-trivial structure of generalized powers in terms of the hypercomplex monogenic variables $z_{k}=x_{k}-x_{0} e_{k}$ together with the fact that for $n>1$ the hypercomplex variable 
$x=x_{0}+\underline{x} \in \mathbb{R}^{n+1}$ and all its powers are not monogenic (cf. [7]) imply a more complicated situation for generalizing these elementary binomial sums to higher dimensions as one naturally would expect. Nevertheless, as it has been shown in [8], [9] there exists for arbitrary dimension $n \geq 1$ a set of special monogenic polynomials $\mathcal{P}_{k}^{n}(x)$ of degree $k=0,1, \cdots$ that preserve almost all properties of the powers of $z$ with positive exponent as one can see in the following list. In particular, they behave like power-law functions under the differentiation operation (properties (i) and (ii) of the list, which characterize a set of Appell polynomials ; see e.g. [10, 11]). In detail, it holds for arbitrary dimension $n \geq 1$

i. $\mathcal{P}_{k}^{n}(x)$ is of exact degree $k$, for each $k=0,1, \cdots$;

ii. $\mathcal{P}_{k}^{n}(x)^{\prime}=k \mathcal{P}_{k-1}^{n}(x)$, for each $k=1,2, \cdots$;

iii. $\mathcal{P}_{0}^{n}(x) \equiv 1$;

iv. $\mathcal{P}_{k}^{n}(0)=0$ for each $k=1, \cdots$.

As usual, this sequence of polynomials is normalized by demanding that $\mathcal{P}_{0}^{n}(x) \equiv 1$ and constructed in such a way that the origin is a common zero of all $\mathcal{P}_{k}^{n}(x)$ with $k>0$. It is evident, that only the use of a hypercomplex derivative enables us to talk about an Appell set in the context of Clifford Analysis. Treating monogenic polynomials exclusively as solutions of a generalized Cauchy-Riemann system would not allow to obtain an analogue to the concept of an Appell set in the real or complex case. The explicit expression of the mentioned polynomials is given by

Theorem 1. Monogenic polynomials of the form

$$
\mathcal{P}_{k}^{n}(x)=\sum_{s=0}^{k} T_{s}^{k}(n) x^{k-s} \bar{x}^{s}, \quad \text { with } \quad T_{s}^{k}(n)=\frac{n !}{(n)_{k}} \frac{\left(\frac{n+1}{2}\right)_{(k-s)}\left(\frac{n-1}{2}\right)_{(s)}}{(k-s) ! s !},
$$

where $a_{(r)}$ denotes the Pochhammer symbol form an Appell set of monogenic polynomials. Let $\nu=$ $\left(\nu_{1}, \cdots, \nu_{n}\right)$ be a multi-index. In terms of generalized powers these polynomials are of the form

$$
\mathcal{P}_{k}^{n}(x)=\mathbf{P}_{k}\left(z_{1}, \cdots, z_{n}\right)=c_{k}(n) \sum_{|\nu|=n} z_{1}^{\nu_{1}} \times \cdots \times z_{n}^{\nu_{n}}\left(\begin{array}{l}
n \\
\nu
\end{array}\right) e_{1}^{\nu_{1}} \times \cdots \times e_{n}^{\nu_{n}},
$$

where

$c_{k}(n):=\frac{k !}{n_{(k)}}\left(\frac{n+1}{2}\right)_{\left(\frac{k-1}{2}\right)} \frac{1}{\left(\frac{k-1}{2}\right) !}, \quad$ if $k \quad$ is odd, $\quad$ and, $\quad c_{k}(n):=\frac{k !}{n_{(k)}}\left(\frac{n+1}{2}\right)_{\left(\frac{k}{2}\right)} \frac{1}{\left(\frac{k}{2}\right) !}$, if $k$ is even.

The structure of the $\mathcal{P}_{k}^{n}(x)$ permits a decomposition in a sum of a real valued part $\widetilde{\mathrm{Sc}}\left(\mathcal{P}_{k}^{n}(x)\right)$ and a product of $\underline{x}$ with another real valued part $\widetilde{\mathrm{Vec}}\left(\mathcal{P}_{k}^{n}(x)\right)$ of $\mathcal{P}_{k}^{n}(x)$, i.e., we can explicitly determine both parts of $\mathcal{P}_{k}^{n}(x)=\widetilde{\mathrm{Sc}}\left(\mathcal{P}_{k}^{n}(x)\right)+\underline{x} \widetilde{\mathrm{Vec}}\left(\mathcal{P}_{k}^{n}(x)\right)$ similar to the decomposition of a complex number. For instance, for the case $n=2$ (here we set $\mathcal{P}_{k}(x):=\mathcal{P}_{k}^{2}(x)$ ) we obtain expressions which for fixed chosen $x_{0}$ and $|\underline{x}|$ are binomial sums:

$$
\begin{aligned}
\widetilde{\mathrm{Sc}}\left(\mathcal{P}_{k}(x)\right) & =\sum_{l=0}^{\left[\frac{k}{2}\right]}\left(\begin{array}{c}
k \\
2 l
\end{array}\right) c_{2 l} x_{0}^{k-2 l}|\underline{x}|^{2 l}(-1)^{l} \\
\widetilde{\mathrm{Vec}}\left(\mathcal{P}_{k}(x)\right) & =\sum_{l=1}^{\left[\frac{k+1}{2}\right]}\left(\begin{array}{c}
k \\
2 l-1
\end{array}\right) c_{2 l-1} x_{0}^{k-(2 l-1)}|\underline{x}|^{2(l-1)}(-1)^{l-1} .
\end{aligned}
$$

\section{The exponential generating function and its application}

Due to the fact that the defining property (ii) of Appell sets implies automatically a direct link to a corresponding exponential function ([11]) and combining this fact with a result of [9] about the relationship to Bessel functions we have the following result. 
Theorem 2. Let $J_{a}(x)$ be Bessel functions of the first kind for orders $a=\frac{n}{2}-1, \frac{n}{2}$. Then

$$
\operatorname{Exp}_{n}(x t)=\widetilde{\operatorname{Sc}}\left(\operatorname{Exp}_{n}\right)+\underline{x} \widetilde{\operatorname{Vec}}\left(\operatorname{Exp}_{n}\right)=\sum_{k=0}^{\infty} \frac{\mathcal{P}_{k}^{n}(x) t^{k}}{k !}
$$

is the exponential generating function of the special monogenic polynomials $\mathcal{P}_{k}^{n}(x)$ and

$$
\begin{aligned}
\widetilde{\mathrm{Sc}}\left(\operatorname{Exp}_{n}\right) & =e^{x_{0} t} \Gamma\left(\frac{n}{2}\right)\left(\frac{2}{|\underline{x}| t}\right)^{\frac{n}{2}-1}\left(J_{\frac{n}{2}-1}(|\underline{x}|)\right), \\
\widetilde{\operatorname{Vec}}\left(\operatorname{Exp}_{n}\right) & =\frac{1}{|\underline{x}|} e^{x_{0} t} \Gamma\left(\frac{n}{2}\right)\left(\frac{2}{|\underline{x}| t}\right)^{\frac{n}{2}-1}\left(J_{\frac{n}{2}}(|\underline{x}|)\right) .
\end{aligned}
$$

Notice, that for $n=2$ the involved Bessel functions are just $J_{0}$ and $J_{1}$ with derivatives very easy to handle. It implies, that also the determination of the generated polynomials in terms of the derivatives of $J_{0}$ and $J_{1}$ is a relatively easy task. And this is just the point for their use in the calculation of binomial sums arising from the comparison with expressions derived from (3) for special values of $x_{0}$ and $|\underline{x}|$. Take, for instance, like in the example at the beginning of Section $3, x_{0}=1$ and $|\underline{x}|=1$. This leads on one side to the binomial sums

$$
\begin{aligned}
& \left(\begin{array}{c}
2 l \\
0
\end{array}\right)-\left(\begin{array}{c}
2 l \\
2
\end{array}\right) \frac{1}{2}+\left(\begin{array}{c}
2 l \\
4
\end{array}\right) \frac{1 \cdot 3}{2 \cdot 4}-\cdots+(-1)^{l}\left(\begin{array}{c}
2 l \\
2 l
\end{array}\right) \frac{(2 l-1) ! !}{(2 l) ! !}=A \\
& \left(\begin{array}{c}
2 l \\
1
\end{array}\right) \frac{1}{2}-\left(\begin{array}{c}
2 l \\
3
\end{array}\right) \frac{1 \cdot 3}{2 \cdot 4}+\cdots+(-1)^{(l-1)}\left(\begin{array}{c}
2 l \\
2 l-1
\end{array}\right) \frac{(2 l-1) ! !}{(2 l) ! !}=B
\end{aligned}
$$

but on the other side to the determination of the corresponding values of the $k$-th coefficients in the well known series development of $J_{0}$ and $J_{1}$ and leads to the value of the binomial sums we are looking for. An algorithm for an easy successive determination of $\widetilde{\mathrm{Sc}}\left(\mathcal{P}_{k}(x)\right)$ and $\widetilde{\mathrm{Vec}}\left(\mathcal{P}_{k}(x)\right)$ has also been developed and shows that the cases $n \geq 2$ are significantly different from the complex case $n=1$.

\section{Acknowledgments}

The research of the first author was partially supported by the Centro de Investigação e Desenvolvimento em Matemática e Aplicações (CIDMA) of the University of Aveiro, through the Portuguese Foundation for Science and Technology (FCT).

\section{References}

[1] D. Z. M. Petkovšek, H. S. Wilf, $A=B$, A. K. Peters, Wellesley, 1996.

[2] D. E. Knuth, The Art of Computer Programming, vol. 1, Addison Wesley, Reading MA, 1968.

[3] H. Wilf, generatingfunctionology, Academic Press, San Diego,, 1994, 2 edn.

[4] O. P. R. L. Graham, D. E. Knuth, Concrete mathematics: a foundation for computer science, AddisonWesley, 1990.

[5] K. Gürlebeck, and H. Malonek, Complex Variables Theory Appl. 39, 199-228 (1999).

[6] H. Malonek, Complex Variables, Theory Appl. 15, 181-191 (1990).

[7] F. Brackx, R. Delanghe, and F. Sommen, Clifford analysis, Pitman, Boston-London-Melbourne, 1982.

[8] H. R. Malonek, and M. I. Falcão, "Special Monogenic polynomials- Properties and Aplications," AIPProceedings, 2007, pp. 764-767. 
[9] M. I. Falcão, and H. R. Malonek, "Generalized Exponentials through Appell sets in $\mathbb{R}^{n+1}$ and Bessel functions," AIP-Proceedings, 2007, pp. 738-741.

[10] P. Appell, Ann. Sci. cole Norm. Sup. 9, 119-144 (1880).

[11] R. P. Boas, and R. C. Buck, Polynomial Expansions of Analytic Functions, Springer, Berlin, 1958. 